\title{
Advantages of Helium and Neon Ion Beams for Intelligent Imaging
}

Huimeng $\mathrm{Wu}^{1}$, Shawn Mcvey ${ }^{1}$, David Ferranti ${ }^{1}$, Chuong Huynh ${ }^{1}$, John Notte ${ }^{1}$, Lewis Stern ${ }^{1}$, Matthew S. Joens $^{2}$, James A. J. Fitzpatrick ${ }^{2}$ and Bernhard Goetze ${ }^{1}$

${ }^{1 .}$ Ion Microscopy Innovation Center, Carl Zeiss Microscopy LLC, One Corporation Way, Peabody MA 01960, USA

2. Waitt Advanced Biophotonics Center, Salk Institute for Biological Studies, 10010 North Torrey Pines Road, La Jolla, CA 92037, USA

Scanning electron microscopy (SEM) has become a crucial tool to visualize the exterior morphology and surface structures for samples in both biology and material science. Conventional SEMs are usually limited by relative low resolution and charging effects. Carbon or metal coating is required for insulator samples to minimize charging effects. However, the coating layer can sometimes obscure miniscule surface details and the coating procedure may generate artifacts, which could be confused with the true ultrastrcuture.

The helium ion microscope (HIM), based on Gas Field Ion Source (GFIS) technology, has a probe size as small as $0.25 \mathrm{~nm}$, which facilitates a new approach to obtain ultra-high resolution images.[1] In particular, the advantages of a HIM over a conventional SEM include substantially higher resolution, greater surface topological details, enhanced material contrast and voltage contrast, a larger depth of field and ion channeling contrast for polycrystalline samples.[2] Besides these, an easy charge control method by using an electron flood gun allows the direct imaging of uncoated biological (or insulator) samples at increasingly high magnification.

The recent development of integrating a neon ion beam into a GFIS column provides a unique opportunity to discover interior morphology and structural information by gradually polishing the surface or actually removing material.[3, 4] $\mathrm{He}$ and $\mathrm{Ne}$ have lower mass than $\mathrm{Ga}$ which allows for a far more precise and controlled nanomachining process. The ability to choose the source ion in one multi-beam ion microscope provides new opportunities to image samples intelligently and allow scientific insights into both the exterior and interior nanoscale structures of samples.

In this presentation, we will discuss in-depth these advantages using $\mathrm{He}$ and $\mathrm{Ne}$ ion beams for imaging and provide examples to further demonstrate these features. The first example is using Ne ion beam to clean a sample to see grain orientations. Fig. 1 shows the images of $\mathrm{Cu}$ structures before and after the Ne ion beam cleaning. The sample was covered by a layer of contamination (containing oxide particles and a carbohydrate layer coating). He ion beam scanning cannot efficiently remove this contamination layer (see Fig. 1a). In contrast, the Ne ion beam was strong enough to polish the sample surface and remove the surface contamination layer. Then the surface grain orientations were clearly shown in both $\mathrm{He}$ and $\mathrm{Ne}$ ion beam images (Fig. $1 \mathrm{~b}$ and $\mathrm{c}$ ). The contrast of Ne ion beam is better than that of He ion beam because of the stronger ion channel effect of $\mathrm{Ne}$ ions. Unlike a gallium focused ion beam (Ga-FIB), a Ne ion beam can precisely control the nanomachining process with minimal beam damage.

The second example is a biological specimen. The goal was to compare HIM imaging with traditional SEM and also study the interior structures of a biological sample using the Ne ion beam milling capability. The resolution attained using HIM imaging is far greater than that obtained from a traditional 
SEM.[5] A Ne ion beam was used to remove the outer skin of a predator nematode. Fig. 2 shows a HIM image after the $\mathrm{Ne}$ ion beam cut. It proved that $\mathrm{Ne}$ ion beam was intense enough to cut through the outer skin of the nematode head without damaging any of the surrounding tissues.[5]

\section{References}

[1] BW Ward, JA Notte and NP Economou, J. Vac. Sci. B. 24 (2006), p. 2871.

[2] L Scipioni, CA Sanford, JA Notte et al, J. Vac. Sci. Tech. B. 27(2009), p. 3250.

[3] MT Postek, AE Vladár and B Ming. AIP Conf. Proc. 1173(2009), p. 249.

[4] M Melli, A Polyakov, D Gargas et al, Nano Letters. 13 (2013), p. 2687.

[5] MS Joens, C Huynh, JM Kasuboski et al, Nature Scientific Reports 3(3514) (2013), p. 1.
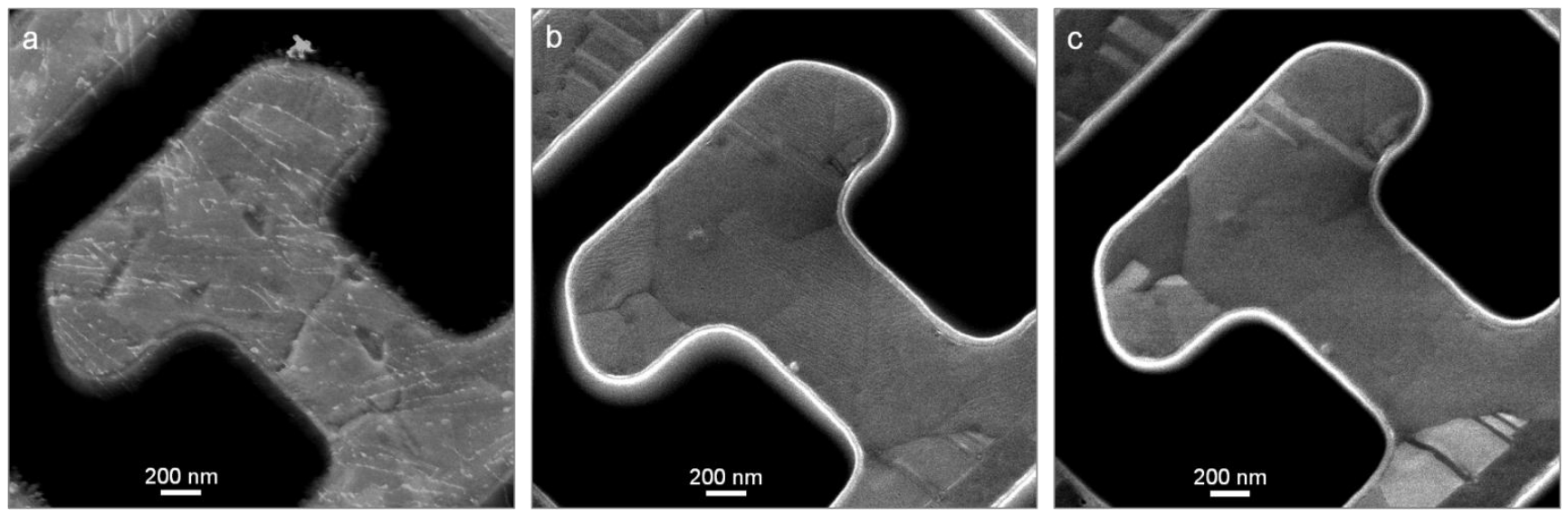

Figure 1. The secondary electron (SE) images of $\mathrm{Cu}$ structures before (a) and after (b, c) Ne ion beam cleaning. (a) and (b) are He ion beam images and (c) is a Ne ion beam image. (c) shows intense grain orientation contrast due to the significant ion channel effect induced by a $\mathrm{Ne}$ ion beam.

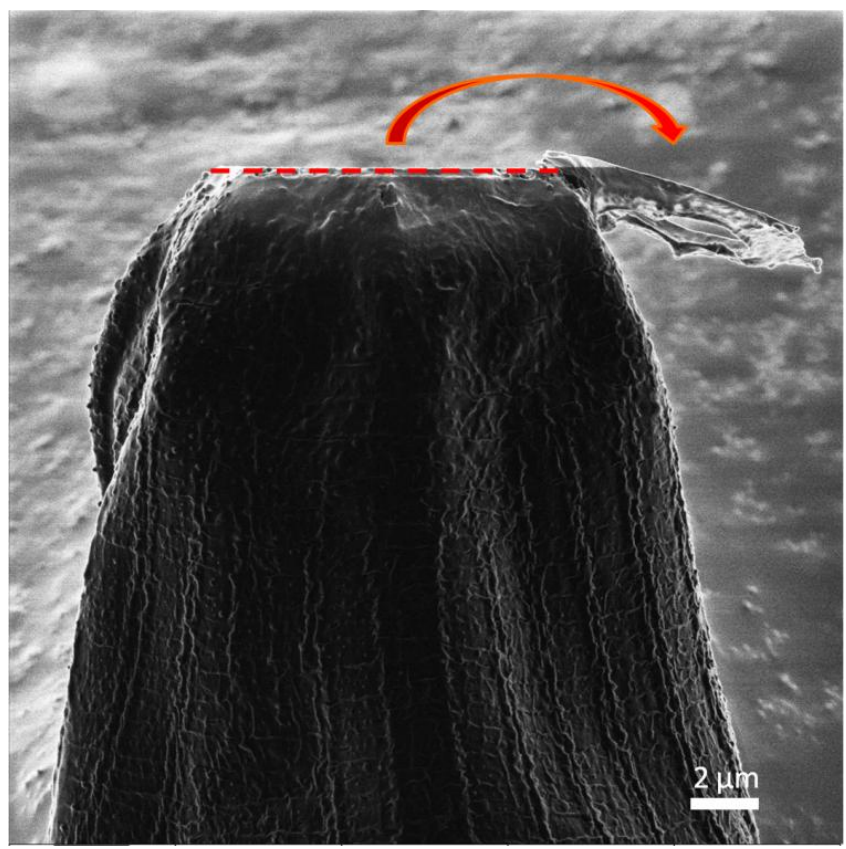

Figure 2. A HIM image of a biological specimen with a Ne ion beam cut through the tip (the red-dashed line is the Ne cut). 